\title{
Assessment of buildings response to earthquakes
}

\author{
A. Farah \\ School of Engineering, Laurentian University, Canada
}

\begin{abstract}
Modern buildings are designed to withstand earthquake loads and to reduce the probability of a disastrous collapse or life-threatening damage due to strong earthquakes. Under medium-intensity earthquakes, the damage to buildings should be repairable, and no building damage or discomfort to the occupants should occur due to minor earthquakes. Buildings are designed to have some structural damage but not collapse due to major earthquakes. The parameters that influence the building behaviour include the characteristics of the earthquake, the soil and the structure itself, such as mass, stiffness and damping. Some earthquake characteristics coupled with suitable site conditions can produce resonance in buildings as was the case that led to the severe damage due to the Mexico earthquake of 1985. In this paper, the response of a building to seismic excitations is evaluated using soil-structure interaction parameters, and its severity is assessed based on the comfort level of the human occupant as judged by the absorbed power (rate of energy dissipation) through a biomechanical model placed on a given floor of the building. The building is represented as a multi-degree-of-freedom system with soil-structure interaction, and the earthquake action is represented by its power spectra. The results indicate that absorbed power differentiates between comfort levels, and therefore human response, at the different floor levels. Results also show that the soil characteristics, the earthquake power spectrum as well as the damping in the structure have significant effect on the response of the building.
\end{abstract}

Keywords: earthquakes, absorbed power, human comfort curves, building serviceability, power spectra, soil-structure interaction. 


\section{Introduction}

Modern buildings are designed to be earthquake-resistant structures that resist minor earthquakes with no damage, moderate earthquakes with no structural but with some non-structural damage, and major earthquakes with some structural and non-structural damage but no building collapse. Emphasis is placed on having life-line buildings such as hospitals, fire stations, nuclear reactors, sewage plants, etc. remain functional after an a major earthquake. These have been the main objectives of earthquake-resistant design for the past four decades (Heidebrecht [1]). Although human comfort is not specified directly in the above, it is expected that such building designs will also be adequate to reduce the probability of occupants suffering intolerable vibrations at least for the weak and low-level moderate earthquakes. Depending on the location and the intensity of the earthquake, the reaction of occupants of building can vary from minor discomfort to panic and fleeing the building. Both wind and earthquakes produce horizontal vibrations in structures that are sensed by the occupants, and although the physical stimulus due to the swaying of buildings is similar in both cases, the psychological impact is normally stronger in the case of earthquakes. This is due to the habituation effect where residents experience and are more familiar with strong winds and storms that have relatively long duration, as compared to those that experience strong earthquakes, usually of short duration. Due to habituation, residents in Japan or California are more likely to tolerate minor to moderate earthquakes as opposed to people in countries considered to be low-risk earthquake zones. Thus, it is important that the human response level to an earthquake be calculated to help assess the impact such an earthquake will have on the human occupant. This will be useful during the design stage to ensure that the serviceability of buildings is satisfactory. The criterion used in this work is the level of absorbed power dissipated through a biomechanical model subject to vibration in the fore-and-aft mode. In order to calculate the absorbed power, the characteristics of the building and the soil as well as the earthquake power spectrum and the parameters of a biomechanical model should be known.

The characteristics of a building for the purpose of dynamic analysis include its stiffness, mass and damping. The latter is important in the design of dynamically sensitive structures where resonance is likely to occur (Jeary [2]). The building shape, type of foundation, the soil properties as well as the ground motion and its duration play an important role. The interaction between the soil and the structure changes the resonant frequencies and the amplitude of the structural vibrations. The soil layers underlying the foundation may have natural periods of vibration coincident or very close to those of the structure and thus may set up resonant motion in the structure. Typical natural periods of soil layers are in the range 0.05 to $1.5 \mathrm{sec}$. As low to medium rise buildings have periods in the range 0.1 to $1.0 \mathrm{sec}$ and tall buildings have periods with a range of 1 to $10 \mathrm{sec}$ ore more, it is quite possible that system resonance may occur due to the periods of the seismic waves coinciding with those of the soil. Such an occurrence is likely to result in serious damage to the building and intolerable vibrations to the occupants or worse should the building collapse as was the case 
in the 1985 Mexico earthquake and the 1995 Hanshin earthquake. To mitigate the likelihood of such an occurrence, design codes require that response analysis be undertaken, at the design stage, for the whole system consisting of the building, foundation and the underlying soil.

\section{Human response and absorbed power}

Occupants of buildings sense vibrations that exceed the threshold of perception and depending on the source and intensity of the vibration, the response can be annoying, discomforting or even intolerable. In the case of vibrations resulting from an earthquake, occupants may panic due to concern over potential serious damage and loss of life. Although other parameters such as amplitude and velocity, coupled with frequency, have been used to indicate the acceptable threshold levels, acceleration is the more frequently used parameter. The commonly accepted acceleration value for the perception threshold is $0.5 \% \mathrm{~g}$, for the threshold of annoyance is $1.5 \% \mathrm{~g}$ and for the threshold of intolerability is $15 \% \mathrm{~g}$, where $\mathrm{g}=9.81 \mathrm{~ms}^{-2}$ is the acceleration due to gravity (Bachmann et al. [3], Chang [4]). However if the recurrence period for disturbing acceleration levels is relatively long, and coupled with well-informed occupants, then such vibration levels may be acceptable. People who have been exposed to high levels of vibration, as would happen during strong windstorms and high magnitude earthquakes, may become more sensitive to vibration in general, even though such vibrations are relatively of low intensity. This suggests that a psychological factor is involved in human response to vibration. Sumitomo et al. [5] studied the rapid increase of complaints by residents after the resumption of rail service about 80 days following the 7.2 magnitude Hanshin earthquake of January 17, 1995. Based on physical measurements and questionnaires, it was concluded that the residents in the stricken area were clearly more affected by the vibration due to the passage of the train, although the measured vibration level was almost the same as before the earthquake. According to the study, the vibration level about which $50 \%$ of the residents in the stricken area complained was $50 \mathrm{~dB}$ as compared to $54 \mathrm{~dB}$ in the non-stricken area. This indicates that a psychological factor was responsible for the difference. This result was explained by the "category judgment method" usually used to determine the psychological reaction to a physical stimulus. Using a constant acceleration level for each of the vibration thresholds implies that such levels are independent of frequency. However, tests on human subjects indicate that this is not the case. Griffin [6] showed that the acceleration for the threshold of perception is frequency dependent. For the standing fore-and-aft mode, the acceleration has a range of 0.02 to $0.09 \mathrm{~ms}^{-2}$ r.m.s. corresponding to the frequency range 2 to $100 \mathrm{~Hz}$, Figure 1. Thus, it is preferable that a criterion that incorporates the characteristics of the vibration, such as absorbed power be used. Absorbed power is a quantity that is computed for a biomechanical model and incorporates the effect of the frequency of vibration. The absorbed power corresponding to the thresholds of human comfort depends on the type of building occupancy such as residential, office, 


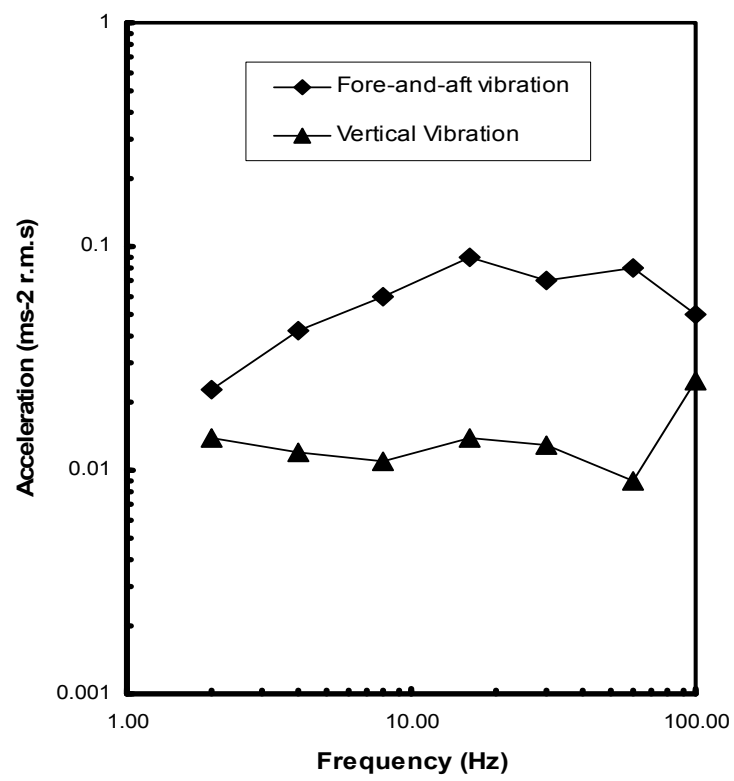

Figure 1: Standing thresholds of vibration in the vertical and fore-and-aft modes [6].

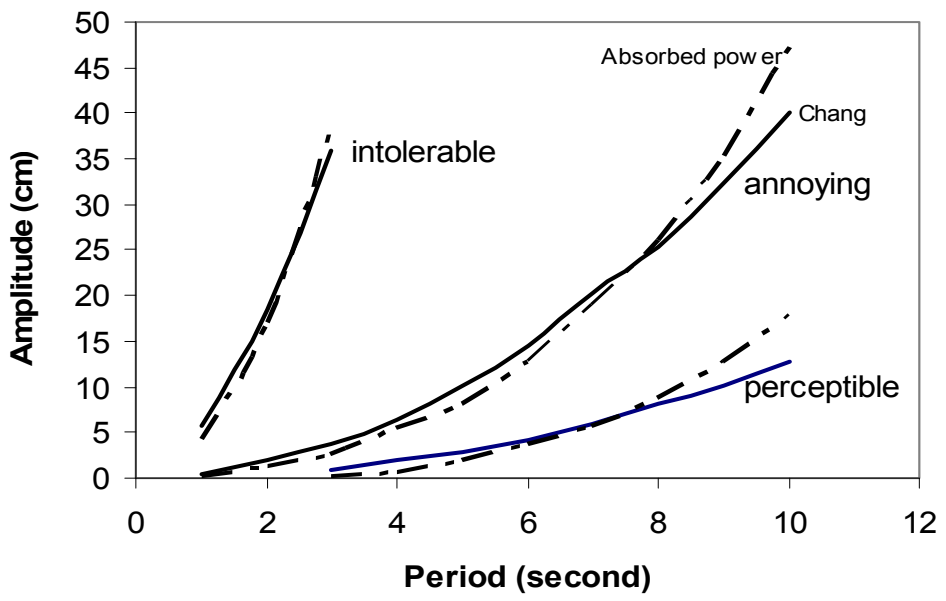

Figure 2: The thresholds of building vibration (Chang [4]), and the levels of absorbed power of $1.33 \times 10^{-4}, 8 \times 10^{-4}$ and $1.7 \mathrm{w}$, corresponding to the thresholds of perception, annoyance and intolerance.

gymnasia etc. Farah [7] showed that the absorbed power values that closely correspond to the published thresholds of perception, annoyance and intolerance are $1.33 \times 10^{-4}, 8 \times 10^{-4}$ and $1.7 \mathrm{w}$ respectively as shown in Figure 2. 


\subsection{Determination of absorbed power}

The calculation of absorbed power requires the modelling of the human body as a biomechanical system, and the determination of the transfer function at the point of contact between the human and the vibrating building (Farah [7]). Absorbed power is the real component of the mechanical power transmitted at the point of contact. The mechanical power transmitted is the vector product of the velocity, $\mathrm{V}$, and the force, F. It is expressed as:

$$
\vec{P}=\vec{F} \times \vec{V}
$$

It can be shown (Farah [8]), that the absorbed power, P, through a biomechanical model can be determined from the integral:

$$
P=-\left\{\int_{0}^{\infty} \omega \operatorname{Im}[G(i \omega)] S_{f}(\omega) d \omega\right\} / \pi
$$

where $\mathrm{G}(\mathrm{i} \omega)$ is the complex transfer function between the force and displacement at the point of contact between the floor and the biomechanical model, and $\mathrm{S}_{\mathrm{f}}(\omega)$ is the floor power spectrum which is related to the power spectrum of the seismic excitation, $\mathrm{S}_{\mathrm{a}}(\omega)$ by:

$$
S_{f}=|H(i \omega)|^{2} S_{a}(\omega)
$$

where $\mathrm{H}(\mathrm{i} \omega)$ is the complex frequency function of the floor level where the biomechanical model is located. Its determination requires that the equations of motion of the structure foundation system be transformed to the frequency domain and the resulting matrix is inverted in closed form. H(i $\omega)$ for each floor level is contained in the inverted matrix (Farah [9]). The inversion process is effected by an efficient procedure based on the Fadeev-Leverrier method. The technique was used as it obtains the inverse of the matrix in closed form without the need to use the normal mode decomposition method. The latter method cannot be used in this situation due to the presence of the coupling terms in the damping matrix resulting from the soil structure interaction. An added benefit to using this inversion procedure is that the determinant of the matrix represents its characteristic equation whose roots give the eigenvalues (frequencies) of the system and its columns provide the eigenvectors (mode shapes) of the structural system.

\subsection{Biomechanical model}

For the absorbed power to be determined, a biomechanical model was developed to represent the human occupant. Models can be developed for a human in the seated, standing or supine position. The vibration can be in the vertical, fore- 
and-aft or lateral mode. The model used in this work is one representing a human in standing position and subject to vibrations in the fore-and-aft mode that simulate motion resulting from wind or seismic action. This model was developed by the author making use of experimental frequency response data by Macduff [10]. The masses of the model components were specified and the stiffness and damping parameters are computed by fitting, in a least square sense, the model frequency response to the experimental data, Figure 3 . It is seen that the data from the fitted model fits closely the experimental data and that there are three prominent whole body vibration frequencies, namely, 0.6, 11.0 and $17.1 \mathrm{~Hz}$ approximately.

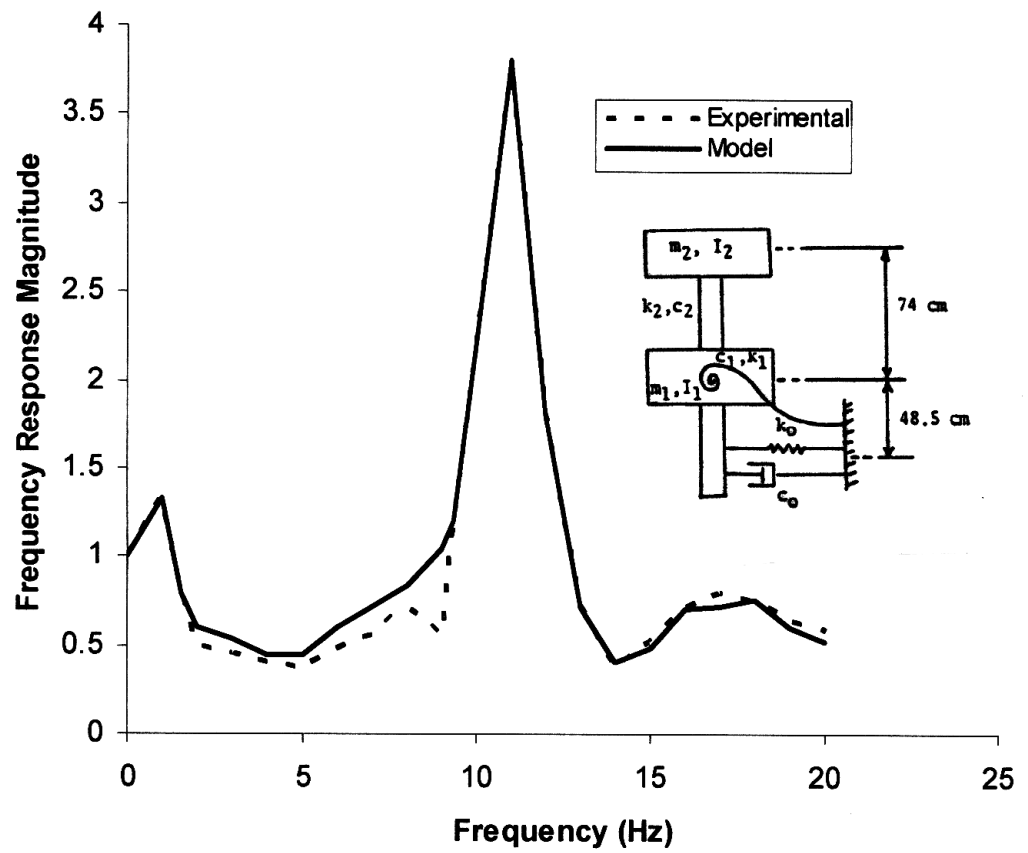

Figure 3: Experimental frequency response data (Macduff [10]), and the corresponding values for the standing model in the fore-and-aft mode.

\section{Soil-structure system and the seismic excitation}

In this study, the soil-structure impedances are represented by equivalent springs and dashpots to simulate the soil stiffness and its damping. The virtual mass of the soil is calculated and added to the mass of the base of the building. While the soil foundation characteristics are usually frequency dependent, these parameters can be taken as frequency independent for engineering applications (Tsai [11]). Here only the translation and rotation (rocking) motions of the foundation are considered. This will result in a soil-structure system having two 
degrees-of-freedom greater than the number of degrees-of-freedom of the structure by itself. The values of the equivalent soil stiffness and damping were calculated by treating the soil as an elastic half-space using the Clough-Penzien formula. The soil parameters used were its density, shear velocity and the radius of the rigid disk representing the foundation half-space. The latter is normally taken as half the width of the building. The equations of motion describing the vibrations of the soil-structure system are [11]:

$$
\left[\begin{array}{cc}
{[M]} & {[0]} \\
{[0]} & {[m]}
\end{array}\right]\{\ddot{v}\}+\left[\begin{array}{cc}
{[C]} & {\left[C_{1}\right]} \\
{\left[C_{2}\right]} & {\left[C_{3}\right]}
\end{array}\right]\{v\}+\left[\begin{array}{cc}
{[K]} & {\left[K_{1}\right]} \\
{\left[K_{2}\right]} & {\left[K_{3}\right]}
\end{array}\right]\{v\}=-\ddot{u}(t)\left\{\begin{array}{c}
\{M\} \\
m_{b} \\
0
\end{array}\right\}
$$

where $[\mathrm{m}]=\left[\begin{array}{cc}m_{b} & 0 \\ 0 & I_{s}\end{array}\right]$ with $\mathrm{m}_{\mathrm{b}}$ being the mass of the base and $\mathrm{I}_{\mathrm{s}}$ represents the sum of the mass moments of inertia of the structure and foundation; [M], [C], and $[\mathrm{K}]$ refer to the mass, damping, and stiffness matrices of the structure respectively and are of size $\mathrm{NxN}$, and $\{M\}$ is the mass vector of the structure. $\left[\mathrm{C}_{1}\right],\left[\mathrm{C}_{2}\right],\left[\mathrm{C}_{3}\right]$ and $\left[\mathrm{K}_{1}\right],\left[\mathrm{K}_{2}\right],\left[\mathrm{K}_{3}\right]$ are damping and stiffness matrices that

couple the structure and the flexible foundation; $u$ is the seismic acceleration and $\{v\}=\left\{\begin{array}{c}\{y\} \\ y_{b} \\ \varphi\end{array}\right\}$ is the displacement vector.

The seismic excitation used is based on records from the 1985 Mexico earthquake (Grigoriu et al. [12]). The SCT-EW acceleration power spectrum shows a peak value at a frequency of $0.5 \mathrm{~Hz}$. Rodriguez [13] points out that $35 \%$ of buildings with 9 to 12 floors suffered significant damage or collapse. The most damaged buildings have periods in the range 1.5 to 2.5 seconds.

\section{Results and discussion}

In order to demonstrate the assessment of the serviceability of buildings subjected to earthquake, an 8-storey building was subjected to the SCT-EW power spectrum of the 1985 Mexico earthquake [12], showing a peak acceleration at about $0.5 \mathrm{~Hz}$. The soil-structure system has a fundamental frequency of $0.51 \mathrm{~Hz}$ corresponding to a soil shear velocity of $400 \mathrm{~m} / \mathrm{s}$. The damping and stiffness of the soil foundation were calculated for several soil shear velocities ranging from 50 to $400 \mathrm{~m} / \mathrm{s}$. The absorbed power value was calculated for the standing biomechanical model placed at each floor. The results indicate that the absorbed power values corresponding to a shear velocity of $400 \mathrm{~m} / \mathrm{s}$ are higher that for the other velocities. Figure 4 shows that the absorbed 
power for the $400 \mathrm{~m} / \mathrm{s}$ shear velocity has a value of $3.75 \mathrm{w}$ which is much higher than the $1.7 \mathrm{w}$ value considered to be the intolerance threshold. Such a level would indicate that the human occupants of such a building would have found the shaking of the building highly intolerable and most probably produced fear and panic. This is due to the $400 \mathrm{~m} / \mathrm{s}$ shear velocity producing a resonance situation as the dominant frequency of the seismic excitation, the fundamental frequency of the soil-structure system and the first whole-body frequency of the biomechanical model are in the range 0.5 to $0.6 \mathrm{~Hz}$.

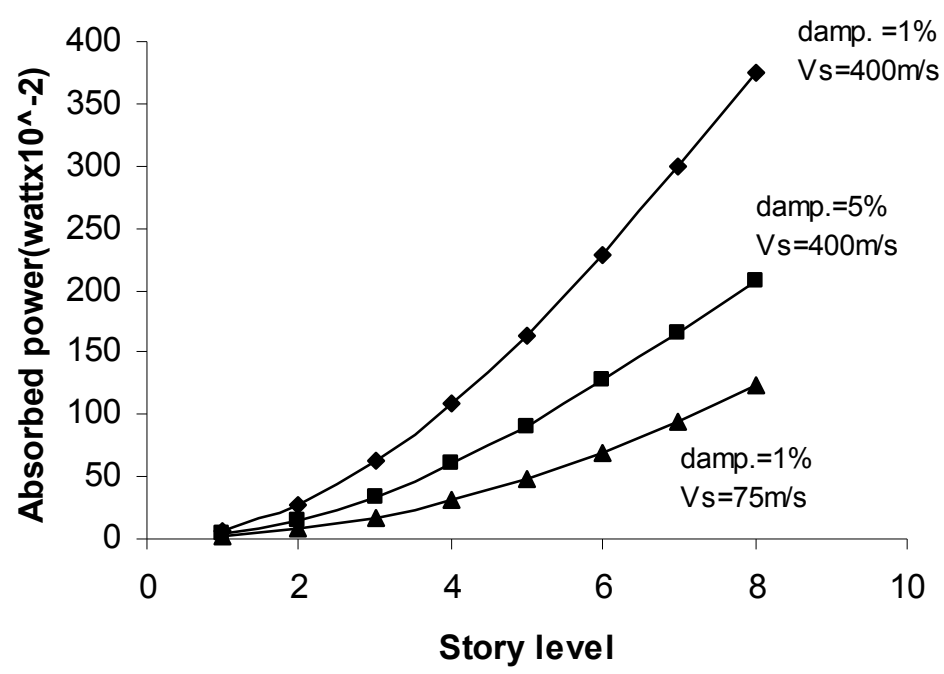

Figure 4: Effect of shear velocity and damping on absorbed power for the 8-storey building (Farah [14]).

The results also show that an increasing the damping from $1 \%$ to $5 \%$ of critical will significantly reduce the value of absorbed power, but is still insufficient to bring the vibrations to a tolerable level. The inter-storey drift corresponding to the $400 \mathrm{~m} / \mathrm{s}$ increases with the floor level but at a decreasing rate as depicted in Figure 5. Also shown is a graph of the ratio of the absorbed power of the floors relative to floor 1 where the absorbed power for the eighth floor is 51 times that of the first floor.

Figure 6 shows the absorbed power for the 8-storey building and the corresponding inter-storey relative absorbed power drift. The maximum relative drift corresponds to the second floor with a value of $283 \%$. The graph also shows that the relative drift decreases with an increase in the floor level reaching a value of $25 \%$. Note that the high values of absorbed power for the relative drift indicate high values of inter-story drift, in terms of displacement. The latter are usually responsible for damage to buildings and should be evaluated at the design stage. 


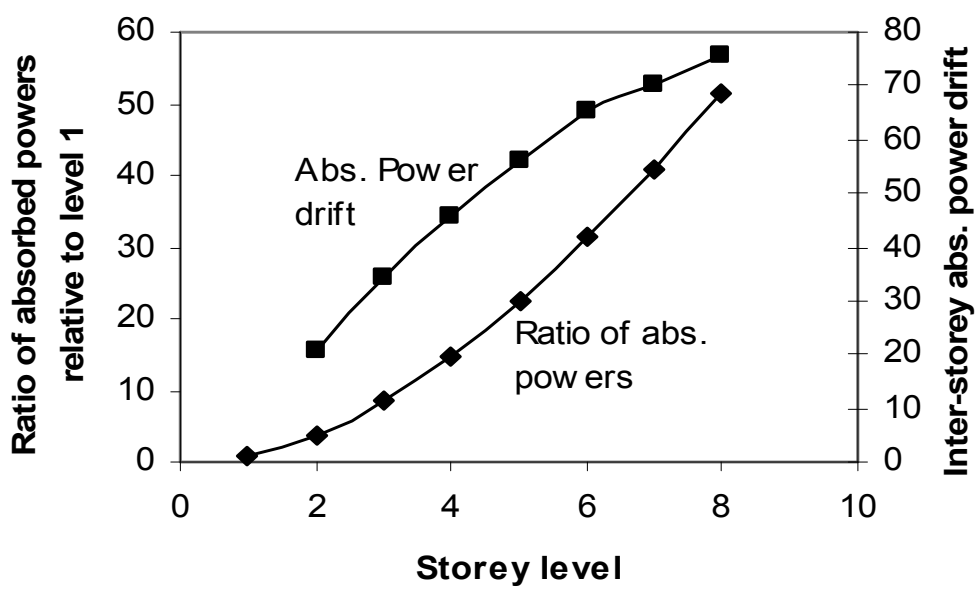

Figure 5: Ratio of absorbed power of floors relative to floor 1, and the interstory absorbed power drift for the 8-storey building for a shear velocity of $400 \mathrm{~m} / \mathrm{s}$.
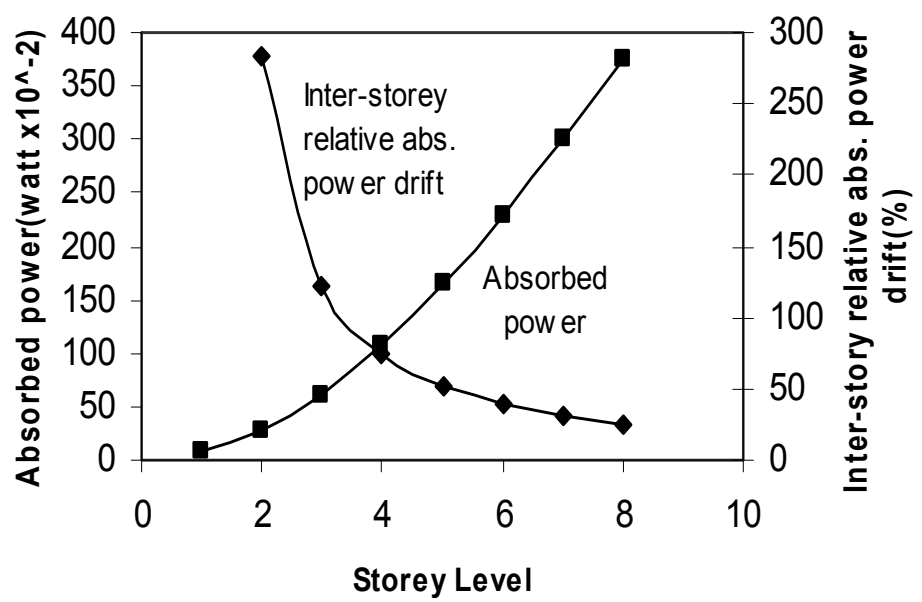

Figure 6: Floor level absorbed power and inter-storey relative drift for the 8storey building for a shear velocity of $400 \mathrm{~m} / \mathrm{s}$.

\section{References}

[1] Heidebrecht, A.C., Earthquake design codes, Proc. of the third Canadian Conf. on Earthquake Engineering, 1, Montreal, pp. 575-608, 1979.

[2] Jeary, A.P., Damping in tall buildings-a mechanism and a predictor. Earthquake Engineering And Structural Analysis,14, pp. 733-750, 1986. 
[3] Bachmann, H., Ammann, W.J., Deischl, F., Vibration Problems In Structures, Springer-Verlag, pp. 77-78, 1995.

[4] Chang, F.K., Human response to motions in tall buildings. Structural Division, ASCE, 99, No. ST6.

[5] Sumitomo, S., Tsujimotos, S., Maeda, S., Kiramura, Y., The influence of the great Hanshin earthquake on human response to environmental vibrations due to the Shinkansen. Industrial Health, 36(3), pp. 290-296, 1998.

[6] Griffin, M.J., Predicting the feeling of vibration in buildings. Proceedings of the Institute of Acoustics, 29(2), 2007.

[7] Farah, A., Structural Serviceability under impact and dynamic load. Proc. of the Ninth Conf. On Structures Under Shock And Impact, eds. N. Jones \& C.A. Brebia, Wit Press: England, pp.467-474, 2006.

[8] Farah, A., Structural serviceability under dynamic loading. ACI Special Publications, SP-86-19, pp.419-434, 1986.

[9] Farah, A., A method for computing the frequency response of a multidegree system. Proc. of the Seventh Canadian Conf. of Applied Mechanics, pp. 903-904, 1979.

[10] Macduff, J.N., Vibrational characteristics of man. Earth moving Industry Conf., Peoria, Illinois, April, 1971.

[11] Tsai, N.C., Modal damping for soil-structure interaction., Engineering Mechanics Division, 100(2), pp.324-341, 1974.

[12] Grigoriu, M., Ruiz, S.E., Rosenblueth, E., Eeri, M., The Mexico earthquake of September 19, 1985-nonstationary models of seismic ground acceleration. Earth quake Spectra, 4(3), pp. 551-567, 1988.

[13] Rodriguez, M., A measure of the capacity of earthquake motions to damage structures. Earthquake Engineering And Structural Dynamics, 23, pp. 627-643, 1994.

[14] Farah, A., Serviceability of buildings subject to seismic excitations. Proc. of the IABSE Symp. On Long-Span and High-Rise Structures, pp. 831836, 1998. 